DOI: $10.2478 /$ lpts-2019-0006

QUANTUM PHYSICS

\title{
ELECTRONIC PROCESSES IN SOLID STATE: DIRAC FRAMEWORK
}

\author{
E. Klotins \\ Institute of Solid State Physics, University of Latvia, \\ 8 Kengaraga Str., Riga, LV-1063, LATVIA \\ klotins@cfi.lu.lv
}

\begin{abstract}
The present paper proposes canonical Dirac framework adapted for application to the electronic processes in solid state. The concern is a spatially periodic structure of atoms distinguished by birth and annihilation of particle states excited due to interaction with the electromagnetic field. This implies replacing the conventional energy-momentum relation specific of the canonical Dirac framework and permissible for particle physics by a case specific relation available for the solid state. The advancement is a unified and consistent mathematical framework incorporating the Hilbert space, the quantum field, and the special relativity. Essential details of the birth and annihilation of the particle states are given by an illustrative two-band model obeying basic laws of quantum mechanics, special relativity, and symmetry principles maintained from the canonical Dirac framework as a desirable property and as a prerogative for the study of the particle coupling and correlation.
\end{abstract}

Keywords: canonical quantization, Dirac field, Hilbert spaces, quasiparticle birth/annihilation

\section{INTRODUCTION}

The long-standing challenge to describe charged particles in solids exposed by electromagnetic fields is how to incorporate the Hilbert space, the quantum field theory, and the special relativity into a consistent unified framework. Current theory for these processes is founded on the study of particle creation. In the original context, the key issue is an electromagnetic field sufficient to create pairs of photoexcited particles from vacuum in high-intensity laser experiments [1], [2]. Though the particle production from vacuum has yet to be experimentally verified, the electronic processes in solids brought benefits from a consistent description of quantum manybody phenomena far from equilibrium. Conventionally, for photoexcited particles created from vacuum in high-intensity laser experiments, we have a solution of the Dirac equation with the classical electromagnetic four-vector potential incorporated. Expanding these two fields in Fourier modes reveals that their wavelengths are much larger than the wavelength of the quantized modes of the Dirac field functions. The 
physical picture is that in this case a photon of the classical four-vector potential does not resolve the quantum nature of the photoexcited electron. It allows the equations of motion for the observables and the slowly varying four-vector fields to be solved self-consistently at the long wavelength scale [3]. However, well-accepted solutions for a free particle and even for quantum fields comprise a quantity identified to the Einstein energy-momentum relation as an element of particle physics and violated in the solids. In this manuscript, we reconsider the energy-momentum relation making it case specific and available for solid-state applications. The viewpoint acquired from the Dirac framework is that physically acceptable solutions imply two mutually consistent equations: the first is Klein-Gordon type related to the Schrödinger equation, and the second is the modified Dirac equation.

Going beyond the canonical Dirac framework, we focus attention on a compound system constituted of the Schrödinger type Hamiltonians. The novelty we claim and the modification we propose is that the equation of motion for this compound system is consistent with the tensor product of its components. Emerging entities are both the expected energy-momentum relation and the (equation of motion for) joint wave function of the compound system.

Ongoing investigations were successful in the oscillator representation for the joint wave function of the compound system [4], [5], and in the mathematical technique for the creation and annihilation operators accepted in this work [6]. In the physics of semiconductors, the excitation of electron-hole pair has attracted much attention and was extensively studied in [7] and [8]. Central in these pioneering approaches is the composite system specified by macroscopic dispersion relations for noninteracting electrons and holes that appear as correlated in the momentum and energy. Charge conjugation of these particles with optical radiation is obtained within the framework of minimal substitution, which the electromagnetic vector potential considered as a macroscopic entity [9].

What we learn from the aforementioned references is that, certainly, the oscillator equation for the composite system is central and plays the role of the Klein-Gordon equation in the canonical Dirac theory, and the canonical Dirac equation, indeed, seems to be consistent with both relativity and even the probability interpretation for a single relativistic particle. What exactly is the challenge, is transition to more general energy-momentum relations spanned by the lattice environment with a variable number of particles. In this context, we give preference to the quantum field functions that a priori imply the Lorentz covariance and the quadratic dispersion of constituting particles. Under these conditions, the Lagrangian density for quantum field functions appears at once, encodes the parameters of macroscopic Hamiltonians, and is available for canonical quantization as desirable properties. This means that component of a composite system is not described independently by its own wave function, without consideration of the state of the other and, generally, arising as a result of interactions between the component degrees of freedom. Finally, as the main result of this work, we obtain a set of differential equations of birth and annihilation operators for an illustrative electron-hole model.

The manuscript is organised as follows. Section 2 presents a general introduction to the problem and some standard information on theory that is used later in the paper. In Section 3, we give details of the modified Dirac equation and the 
associated quantum field functions. In Section 4, the Lagrangian density is built from the modified Dirac equation and available for the minimum action mathematical technique. Finally, Section 5 summarises the results of this study. We use natural units, $\hbar=c=1$ restoring them explicitly where adding insight.

\section{BASIC CONCEPTS AND DEFINITIONS}

While the basis of quantum mechanics is provided through purely algebraic relations, for specific results a complementary representation of the Hilbert space, the quantum field theory and the special relativity are used. To bring rationale of all, a special attention is paid to the quantum mechanical wave functions defined so that the Hilbert space is given by a set of elements $H=(|\psi\rangle,|\varphi\rangle,|\chi\rangle, \ldots)$ obeying postulates for scalar product, linearity, completeness, and separability. This is definitely the case of Schrödinger type wave functions for Hamiltonians comprising both the kinetic and time independent potential energy terms. For arbitrary dimensionality, the joint wave function implies linearly independent vector spaces $V=\left\{v_{1}, v_{2}, v_{3}\right\}$ and $W=\left\{w_{1}, w_{2}, w_{3}\right\}$, where $\left\{\vec{e}_{1}, \vec{e}_{2}, \ldots, \vec{e}_{n}\right\}$ is the basis vectors of $V$, and similarly $\left\{\vec{f}_{1}, \vec{f}_{2}, \ldots, \vec{f}_{m}\right\}$ for $W$. The tensor product of the vector spaces $V \otimes W$ is spanned by the basis vectors $\vec{e}_{i} \otimes \vec{f}_{j}$ as given by relation

$$
\vec{v} \otimes \vec{w}=\left(\sum_{i}^{n} v_{i} \vec{e}_{i}\right) \otimes\left(\sum_{j}^{m} w_{j} \vec{f}_{j}\right)=\sum_{i}^{n} \sum_{j}^{m} v_{i} w_{j}\left(\vec{e}_{i} \otimes \vec{f}_{j}\right) .
$$

We categorize this property as a fundamentally new multi-particle approach available for the creation and annihilation of particle states in far-from-equilibrium conditions.

Consequences for the Dirac framework [10] are that instead of the prototypical Klein-Gordon equation for a free relativistic particle we have the oscillator equation for the case specific energy-momentum relation. The associated Dirac equations for spinor functions turn into a modified Dirac equation distinguished by first order in space and time derivatives and by numerical coefficients connecting it with the oscillator equation. Requested consistence between both equations is fully defined by drawing comparison between their differential operators of appropriate order. Necessary restrictions of the special relativity are encoded in appropriate quantum field functions. This allows the Lagrange and Hamilton transition from the joint wave function of the composite system to the Lagrangian density for the canonically quantized field operators of the Hamiltonian density and the methods of quantum statistics available.

The observable quantities emerge in a standard way as integrals over timedependent distribution functions, however, being beyond the scope of this manuscript. 


\section{BUILDING THE MODIFIED DIRAC EQUATION}

In this section, we follow the canonical Dirac framework distinguished by the wave equation that is first order in both space and time derivatives and linear in the momentum

$$
(\hat{E}-\bar{\alpha} \cdot \vec{p}-\beta m) \Psi(x)=0,
$$

where, conventionally, solutions for the entities $\bar{\alpha}$ and $\beta$ imply drawing a comparison between the Dirac equation (2) and the Klein-Gordon equation [11].

The concern is the momentum-energy relation of composite systems given implicitly by the tensor product mathematical technique. As an example, we present a two-band model specified by the conduction and valence states as well as by width of the band gap. We set the Dirac equation (2) modified for the electron and the hole as

$$
\left(i \hbar \frac{\partial}{\partial t}+i \hbar \bar{\alpha} \cdot \vec{\nabla}+i \hbar \bar{\gamma} \cdot \vec{\nabla}-\beta \frac{\Delta}{2}\right) \Psi(x)=0 .
$$

Here the constraints on the constants $\bar{\alpha}, \bar{\gamma}$ and $\beta$ follow from drawing a comparison between the (i) extended Dirac equation (3) squared and (ii) the oscillator equation.

We build the constituting equations for the prototypic composite system making use of the macroscopic constituting Hamiltonians $H_{c}$ and $H_{v}$ as

$$
H_{c / v}= \pm \mathbf{p}^{2} /\left(2 m_{e}\right) \pm \Delta / 2
$$

where the momentum $\mathbf{p}$ and the (electronic) band gap $\Delta$ are classical quantities. Quantization of classical dynamic quantities implies the energy operator $\hat{E} \rightarrow i \mathrm{~h} \partial_{t}$ and the momentum operator $\hat{\mathbf{p}} \rightarrow-i \mathrm{~h} \nabla$. Finally, the equation of motion for this composite system (oscillator equation) is built up by tensor product (1) at $m=n=3$ as

$$
(\stackrel{\mathrm{r}}{v} \otimes \stackrel{\mathrm{r}}{w}) \Psi(\mathbf{x}, t)=0
$$

Treatment of the tensor product implies components of the vector spaces $V$ and $W$ in the operator representation as $v=\hat{E}_{c}-\Delta / 2-\varepsilon_{c}(\hat{\mathbf{p}})$ and $w=\hat{E}_{v}+\Delta / 2+\varepsilon_{v}(-\hat{\mathbf{p}})$ gives the expected oscillator equation for the composite system as

$$
\left(-\frac{\Delta^{2}}{4}+\hat{E}^{2}-\frac{\Delta}{2} \varepsilon_{c}(\hat{\mathbf{p}})-\hat{E} \varepsilon_{c}(\hat{\mathbf{p}})-\frac{\Delta}{2} \varepsilon_{v}(-\hat{\mathbf{p}})+\hat{E} \varepsilon_{v}(-\hat{\mathbf{p}})-\varepsilon_{c}(\hat{\mathbf{p}}) \varepsilon_{v}(-\hat{\mathbf{p}})\right) \Psi(\mathbf{x}, t)=0
$$




\subsection{SQUARED REPRESENTATION OF THE MODIFIED DIRAC EQUATION}

In this subsection, we are drawing comparison between the Dirac equation (4) squared and the oscillator equation (6) resulting in the relations for the coefficients $\alpha, \gamma$ and $\beta \quad$ as $\alpha_{x}^{2}=\alpha_{y}^{2}=\alpha_{y}^{2}=-\Delta /\left(4 m_{e}\right), \quad \gamma_{x}^{2}=\gamma_{y}^{2}=\gamma_{z}^{2}=-\Delta /\left(4 m_{h}\right) \quad$ and $\beta^{2}=1$. The mixed projections of $\bar{\alpha}$ and $\bar{\gamma}$ satisfy anticommutation relations $\alpha_{j} \alpha_{k}+\alpha_{k} \alpha_{j}=0$ and $\gamma_{j} \gamma_{k}+\gamma_{k} \gamma_{j}=0$ at $j, k=x, y, z, j \neq k$. The anticommutation relations of $\bar{\alpha} \beta$ type is $\left(\beta \alpha_{j}+\alpha_{j} \beta\right)=0 ; j=x, y, z$ and of $\bar{\gamma} \beta$ type is $\left(\beta \gamma_{j}+\gamma_{j} \beta\right)=0 ; j=x, y, z$. These anticommutation relations imply that at least for the illustrative two-band model the classical Dirac $\bar{\alpha}$-matrices given by relation $\alpha_{x}^{2}=\alpha_{y}^{2}=\alpha_{z}^{2}=\mathbf{1}$ and supporting anticommutation conditions in the canonical Dirac framework are violated. Instead, the anticommutation conditions must be encoded in the appropriate quantum field function(s).

The same concern is for the $\bar{\gamma}$-factor in (3). The consequences for the mixed projections of $\overline{\alpha \gamma}$ are that all of it turns to zero and the similarity with canonical anticommutation relations [11] is definitely formal. As a result, the full solution of (11) implies explicit relations $\bar{\alpha}=\sqrt{-\Delta /\left(4 m_{e}\right)}, \bar{\gamma}=\sqrt{-\Delta /\left(4 m_{e}\right)}$.

The unknown yet overall wave function must obey the Fourier mode expansion as

$$
\Psi(x)=\int d p^{4}(2 \pi)^{-4} \tilde{\Psi}(p) \exp (-i p x),
$$

where properties of the composite system are encoded in the momentum space field $\widetilde{\Psi}(p)$ and the 4-vector scalar product $p x=E t / \hbar-\mathbf{p} \cdot \mathbf{x} / \hbar$ is in the $\{+,-,-,-\}$ metric signature. In full detail, the Dirac equation (3) is written in differential operators as

$$
\begin{aligned}
& -\frac{\Delta}{2} \Psi+i \hbar \Psi_{t}+\left(i \hbar \alpha_{z} \Psi_{z}+i \hbar \alpha_{y} \Psi_{y}+i \hbar \alpha_{x} \Psi_{x}\right) \\
& +\left(i \hbar \gamma_{z} \Psi_{z}+i \hbar \gamma_{y} \Psi_{y}+i \hbar \gamma_{x} \Psi_{x}\right)=0
\end{aligned}
$$

where the spatial gradient terms are included in brackets. Finally, implementing (7) in (8) turns the equation of motion for the overall quantum wave function of composite system as

$$
\frac{\Delta}{2} \Psi-\left(\sqrt{2 \Delta \varepsilon_{e}(\mathbf{p})}+\sqrt{2 \Delta \varepsilon_{h}(-\mathbf{p})}\right) \Psi+i \hbar \dot{\Psi}=0 .
$$

\subsection{QUANTUM FIELD FUNCTIONS}

Extensions of (9) to more realistic systems interacting with external fields and exhibiting variable number of excited states require transition from quantum field functions to the quantum operator functions. The search is, first, the Lorentz invariance 
[12] and the four Fourier representations of field functions related to positive (conduction) and negative (valence) energy states and, second, harmonization of the relativistic invariant integration measure with the macroscopic dispersion relations of the electrons and holes. To this end, we apply the Lorentz invariant representation for quantum field functions as [12].

$$
\begin{aligned}
& \Psi(x)=\int d^{4} p(2 \pi)^{-4} \tilde{\Psi}(p) \exp (-i p x), \\
& \Psi^{*}(x)=\int d^{4} p(2 \pi)^{-4} \tilde{\Psi}^{*}(p) \exp (i p x),
\end{aligned}
$$

where after (1), (2) the momentum space field is given by expansion in $\delta-$ functions as

$$
\begin{aligned}
& \tilde{\Psi}(E, \mathbf{p}) \rightarrow \\
& \delta\left[E-\frac{\Delta}{2}-\varepsilon_{c}(\mathbf{p})\right]\left[E+\frac{\Delta}{2}+\varepsilon_{v}(-\mathbf{p})\right] \psi(E, \mathbf{p})=\delta g(E, \mathbf{p}) \psi(E, \mathbf{p}) \\
& \tilde{\Psi}^{*}(E, \mathbf{p}) \rightarrow \\
& \delta\left[E-\frac{\Delta}{2}-\varepsilon_{c}(\mathbf{p})\right]\left[E+\frac{\Delta}{2}+\varepsilon_{v}(-\mathbf{p})\right] \psi^{*}(E, \mathbf{p})=\delta g(E, \mathbf{p}) \psi^{*}(E, \mathbf{p})
\end{aligned} .
$$

Equations (12),(13) support the necessary connection between the macroscopic parameters of the compound system and the quantum field functions (10), (11).

Optical radiation implies a spatially homogeneous time-dependent electric field, represented (at appropriate wavelength) by the vector potential in the Coulomb gauge $\nabla \cdot \mathbf{A}=0$. In full, the momentum space field contributes as a replacement $\mathbf{p} \rightarrow \mathbf{p} \pm \mathbf{A}(t)$ in quantum field functions, in the Lagrangian density, and, finally, in the birth and annihilation operators of associated quasiparticles, here - electrons and holes. However, this desirable property involves complexities, and, in this illustrative example, we restrict the calculations in free field mode, restating the effect of timedependent electric field in the final relations.

Going over the technicalities for connection between the macroscopic parameters of the compound system and the quantum field functions (10), (11) we write the expected field functions decomposed in sums over momentum $\mathbf{p}$

$$
\begin{aligned}
& \Psi(\mathbf{x}, t)=\frac{1}{L^{3 / 2}} \sum_{\mathbf{p}}\left(\frac{1}{\sqrt{\Delta+\varepsilon_{c}(\mathbf{p})+\varepsilon_{v}(-\mathbf{p})}}\left(a_{e, \mathbf{p}} e^{-i\left(\frac{\Delta}{2}+\varepsilon_{c}(\mathbf{p})\right) t}+a_{h,-\mathbf{p}} e^{i\left(\frac{\Delta}{2}+\varepsilon_{v}(-\mathbf{p})\right) t}\right)\right) e^{i \mathbf{p x}} \\
& \Psi^{*}(\mathbf{x}, t)=\frac{1}{L^{3 / 2}} \sum_{\mathbf{p}}\left(\frac{1}{\sqrt{\Delta+\varepsilon_{c}(\mathbf{p})+\varepsilon_{v}(-\mathbf{p})}}\left(a_{c, \mathbf{p}}^{*} e^{+i\left(\frac{\Delta}{2}+\varepsilon_{c}(\mathbf{p})\right) t}+a_{v,-\mathbf{p}}^{*} e^{-i\left(\frac{\Delta}{2}+\varepsilon_{v}(-\mathbf{p})\right) t}\right)\right) e^{-i \mathbf{p x} \mathbf{x}}
\end{aligned}
$$




\section{LAGRANGE AND HAMILTON FORMALISM}

The Lagrange and Hamilton formalism implies mathematical abstractions regarded to the fundamentals of quantum electrodynamics, whereas on physical grounds the expectations is to describe evolution of the particle distribution functions during the external field evolution. Having the Lorentz invariant quantum field functions (10), (11) we accept that sufficient condition for the Lagrangian density to have physical sense is to return the modified Dirac equation (8), (9). To this end we set the Lagrangian density for the quantum field functions (14), (15) as

$$
\mathcal{L}=\frac{\Delta}{2} \Psi \Psi^{*}-\left(\sqrt{2 \Delta \varepsilon_{e}(\vec{p})}+\sqrt{2 \Delta \varepsilon_{h}(-\vec{p})}\right) \Psi \Psi^{*}+i \hbar \Psi^{*} \dot{\Psi}-i \hbar \dot{\Psi}^{*} \Psi+\frac{\hbar^{2}}{\Delta} \dot{\Psi}^{*} \dot{\Psi}
$$

The canonically conjugate momentum fields read in usual way as

$$
\begin{aligned}
& \pi(\vec{x}, t):=\frac{\partial \mathcal{L}}{\partial \dot{\Psi}(\vec{x}, t)} \rightarrow \sum_{\mathbf{p}}\left(i \hbar \Psi^{*}+\frac{\hbar^{2}}{\Delta} \dot{\Psi}\right), \\
& \pi^{*}(\vec{x}, t):=\frac{\partial \mathcal{L}}{\partial \dot{\Psi}^{*}(\vec{x}, t)} \rightarrow \sum_{\mathbf{p}}\left(-i \hbar \Psi+\frac{\hbar^{2}}{\Delta} \dot{\Psi}\right),
\end{aligned}
$$

and the free field Hamiltonian density is given by

$$
\begin{aligned}
& H_{\text {tot }}=\sum_{\mathbf{p}}\left(\pi(\vec{x}, t) \dot{\Psi}+\pi^{*}(\vec{x}, t) \dot{\Psi}^{*}-\mathcal{L}\right) \rightarrow \\
& \sum_{\mathbf{p}}\left(\left(-\frac{\Delta}{2}+\sqrt{2 \Delta \varepsilon_{e}(\vec{p})}+\sqrt{2 \Delta \varepsilon_{h}(-\vec{p})}\right) \Psi \Psi^{*}+\frac{\hbar^{2}}{\Delta} \dot{\Psi} \dot{\Psi}^{*}\right)
\end{aligned}
$$

Implementation of the quantum field functions (14), (15) in (19) gives the free field Hamiltonian density in quasiparticle representation as

$$
H_{t o t}=\frac{1}{V} \sum_{\mathbf{p}}\left(\begin{array}{l}
\frac{\Delta \varepsilon_{c}(\mathbf{p})+\varepsilon_{c}(\mathbf{p})^{2}-\left(\frac{\Delta}{2}\right)^{2}+\sqrt{2 \Delta \varepsilon_{e}(\mathbf{p})}+\sqrt{2 \varepsilon_{v}(-\mathbf{p})}}{\Delta+\varepsilon_{c}(\mathbf{p})+\varepsilon_{v}(-\mathbf{p})} a_{e}(\mathbf{p}) a_{e}^{*}(\mathbf{p})+ \\
\frac{\Delta \varepsilon_{v}(-\mathbf{p})+\varepsilon_{v}(-\mathbf{p})^{2}-\left(\frac{\Delta}{2}\right)^{2}+\sqrt{2 \Delta \varepsilon_{e}(\mathbf{p})}+\sqrt{2 \varepsilon_{v}(-\mathbf{p})}}{\Delta+\varepsilon_{c}(\mathbf{p})+\varepsilon_{v}(-\mathbf{p})} a_{h}(-\mathbf{p}) a_{h}^{*}(-\mathbf{p})
\end{array}\right)
$$


that is available for the minimal action treatment. The factors before the creation and annihilation operators in (20) are functions of the macroscopic parameters of the compound system as desirable property.

The necessary equations of motion for the creation and annihilation operators, implies the quantum field functions (14), (15) in the canonical quantized fashion as

$$
\begin{aligned}
& \Psi(\mathbf{x}, t)=\frac{1}{\sqrt{V}} \sum_{\mathbf{p}}\left(\frac{\sqrt{\Delta}}{\sqrt{\Omega(\mathbf{p}, t)}}\left(a_{e}(\mathbf{p}, t)+a_{h}(-\mathbf{p}, t)\right)\right) e^{i \mathbf{p} \mathbf{x}}, \\
& \Psi^{\dagger}(\mathbf{x}, t)=\frac{1}{\sqrt{V}} \sum_{\mathbf{p}}\left(\frac{\sqrt{\Delta}}{\sqrt{\Omega(\mathbf{p}, t)}}\left(a_{c}^{\dagger}(\mathbf{p}, t)+a_{v}^{\dagger}(-\mathbf{p}, t)\right)\right) e^{-i \mathbf{p x}} .
\end{aligned}
$$

Then, going back to the canonically conjugate momentum fields (17), (18) we turn to the standard definition

$$
\mathcal{L}=\pi \dot{\Psi}+\pi^{*} \dot{\Psi}^{*}-H_{t o t}
$$

where the Lagrangian density (23) is consistent with the integrand of the minimal action relation $S=\int d x \mathcal{L}$ and the equations of motion are given by functional derivatives over the time dependent amplitudes $a_{e, h}(\mathbf{p}, t)$.

Parameters of the compound system are encoded by factor $\Omega(\mathbf{p}, t)=\Delta+\varepsilon_{c}(\mathbf{p}, t)+\varepsilon_{v}(-\mathbf{p}, t)$ and the dispersions are given by (3), (4). Similarly to [7], extensions of the free field relations to the case of electromagnetic radiation is obtained by the replacement $\mathbf{p} \rightarrow \mathbf{p} \pm \mathbf{A}(t)$ in quantum field functions (21, (22).

\section{CONCLUSIONS}

In this manuscript, the canonical Dirac framework is adapted for application to the electronic processes in solid state. The advancement is in replacing the conventional energy-momentum relation permissible for particle physics by a case specific relation available for solid-state applications. As a result, a complementary representation of the Hilbert space, the quantum field theory and the special relativity are applied. Emerging entities are both the expected case specific energy-momentum relation and the (equation of motion for) joint wave function of the compound system of particles. We claim that this result is original, previously unpublished, and from the best of our knowledge no such modification of Dirac theory is published elsewhere. Because of application to solid state and as an illustration of the proposed modification of the canonical Dirac framework, the electron-hole pair is discussed in full detail.

While conventionally we are concerned with fermionic particles, the modified Dirac framework describes fields on a level where the particles are not there from 
the start. It is only if to solve the field equations in presence of a pulse of external field that gives rise to the emergence of quasiparticles. This desirable property is a prerogative for strong coupling and correlation approaches having come to the fore of modern condensed matter physics.

\section{ACKNOWLEDGEMENTS}

The present research has been supported by the Institute of Solid State Physics, the University of Latvia in the frames of the ERA-NET HarvEnPiez project (No. ES RTD/2016/15).

\section{REFERENCES}

1. Di Piazza, A., Müller, C. K., Hatsagortsyan, Z., \& Keitel, C. H. (2012). Extremely highintensity laser interactions with fundamental quantum systems. Rev. Mod. Phys., 84, 1177.

2. Poder, K., Tamburini, M., Sarri, G., Di Piazza, A., Kuschel, S., Baird, C. D., \& Zepf, M. (2018). Experimental signatures of the quantum nature of radiation reaction in the field of an ultraintense laser, Phys. Rev. X 8, 031004.

3. Hegelich, B. M., Labun, L., \& Labun, O. Z. (2017) Finding quantum effects in strong classical potentials. J.Plasma Phys., 83(3), 595830301.

4. Pervushin, V.N., \& Skokov, V.V. (2006). Kinetic description of fermion production in the oscillator representation, Acta Physica Polonica B, 37(9), 2587-2600.

5. Pervushin, V. N., Skokov, V. V., Reichel, A. V., Smolyansky, S. A., \& Prozorkevich, A. V. (2005). The kinetic description of vacuum particle creation in the oscillator representation. International Journal of Modern Physics A, 20(24), 5689-5704.

6. Friesen, A.V., Prozorkevich, A.V. , Smolyansky, S.A., \& Bonitz, M. (2007). Nonperturbative kinetics of electron-hole excitations in strong electric field. In Saratov Fall Meeting 2006: Laser Physics and Photonics, Spectroscopy and Molecular Modeling VII, eds. V.L. Derbov, L.A. Melnikov, and L.M. Babkov, Proc. SPIE 6537, article id. 653707.

7. Smolyansky, S.A., Bonitz, M., \& Tarakanov, A.V. (2010). Strong field generalization of the interband transitions kinetics. Physics of Particles and Nuclei, 41(7), 1075-1078.

8. Smolyansky, S. A., Panferov, A. D., Blaschke, D. B., Juchnowski, L., Käampfer, B., \& Otto, A. (2017). Vacuum particle-antiparticle creation in strong fields as a field induced phase transition. Russ. Phys. J., 59(11), 1731-1738.

9. Panferov, A. D., Smolyansky, S. A., Titov, A. I., Kaempfer, B., Otto, A., Blaschke, D. B., \& Juchnowski, L. (2017). Field induced phase transition in the few photon regime, EPJ Web Conf., 138, 07004.

10. Dirac, P. (1928). The quantum theory of the electron. Proc. R. Soc. Lond., A117, 610634.

11. Thomson, M. (2013). Modern particle physics. Cambridge: Cambridge University Press.

12. Álvarez-Gaumé,L., \& Vázques-Mozo, M.Á.(2012). An invitation to quantum field theory. Lecture Notes in Physics 839. Springer-Verlag Berlin Heidelberg. DOI:10.1007/978-3642-23728-7_2 


\section{ELEKTRONU PROCESI CIETĀ VIELĀ: DIRAKA IETVARS}

\section{E. Klotiņš}

\section{Kopsavilkums}

Raksts veltîts kanoniskās Diraka teorijas modifikācijai elektronu procesu aprakstam cietā vielā. Nepieciešamā pāreja no Diraka daļiņu fizikas uz fotoierosinātu elektriski lādētu dalinu kinētiku cietā vielā izveidota kā matemātiska struktūra, kurā ietilpst savstarpēji saistīti Hilberta telpas, kvantu lauka, un speciālās relativitātes pielietojumi. Attiecīgā fizikālā situācija ietver telpiski periodisku atomāru struktūru, kuru raksturo daļiņu dzimšana un anihilācija elektromagnētiskā lauka ietekmē. Detalizēta daļiņu dzimšana un anihilācija ilustrēta, izmantojot divu zonu modeli, kurā saglabāti arī kanoniskai Diraka teorijai raksturīgie kvantu mehānikas, speciālās relativitātes un simetrijas principi kā nepieciešamais priekšnoteikums daļiņu mijiedarbības un korelācijas kvantu kinētiskam aprakstam.

02.12.2018. 